\title{
SWAP and LYRA: Space Weather from a small spacecraft
}

\author{
J.M. Defise', J.H. Lecat', Y. Stockman', P. Rochus ${ }^{1}$, E. Mazy ${ }^{1}$, F. Denis', J.P. Halain ${ }^{1}$, L. Rossi ${ }^{1}$, T. Thibert', D. Berghmans ${ }^{2}$, \\ J.F. Hochedez ${ }^{2}$, N. Bogdan', A. Ben Moussa ${ }^{2}$, G. Lawrence ${ }^{2}$, T. Katsiyannis ${ }^{2}$, W. Schmutz ${ }^{3}$, S. Koller ${ }^{3}$, U. Schühle', \\ K. Haenen ${ }^{5}$, P. Gloesener ${ }^{6}$, V. Thomas ${ }^{6}$ \\ ${ }^{1}$ Centre Spatial de Liège (CSL) - University of Liège - Av. Pré Aily, 4031 Angleur - Belgium, jmdefise@ulg.ac.be \\ ${ }^{2}$ Royal Observatory of Belgium (ROB) - 3 -Av. Circulaire, 1180 Brussels - Belgium \\ ${ }^{3}$ Physikalisch-Meteorologisches Observatorium Davos - Switzerland \\ ${ }^{4}$ Max Planck Institut für Sonnensystemforschung - Lindau - Germany \\ ${ }^{5}$ IMOMEC -Diepenbeek - Belgium \\ ${ }^{6}$ AMOS SA - Angleur - Belgium
}

\begin{abstract}
Two scientific instruments for Sun observations are being developed to be part of the payload of the ESA's second microsatellite, Proba-II (Project for On-board Autonomy). PROBA-2 is scheduled for launch in early 2007, on a low earth orbit. Like Proba-1, in orbit since October 2001, Proba-2 is a 100-kilogram class spacecraft.

PROBA-II will demonstrate new advanced technologies on its scientific payload but also on new platform subsystems such as star tracker, digital Sun sensor, cool gas generator, solar array concentrator, Li-Ion Battery, new central processor, ...

This paper is dedicated to the solar payload, comprising the $S$ un Watcher using Active Pixel System detector and inage Processing (SWAP) and the Lyman alpha Radiometer (LYRA), both aiming at Sun observations.

SWAP, the Belgian-led main instrument, will continuously provide detailed images of the solar atmosphere, by the light of extreme ultraviolet rays, at $17.4 \mathrm{~nm}$, completely absorbed by the terrestrial atmosphere.
\end{abstract}

\section{INTRODUCTION: THE PROBA-2 MISSION}

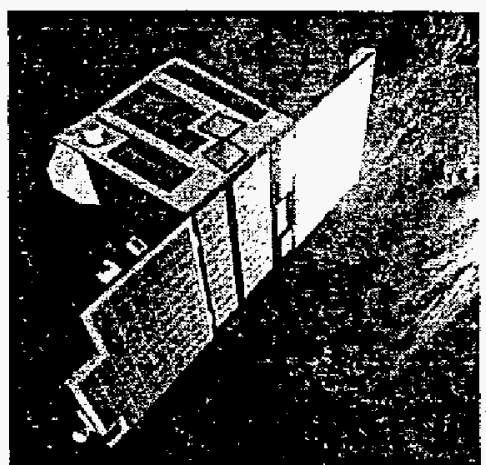

Figure t. Rendering of PROBA-2 (courtesy Verhaert N.V.)

PROBA-2 is a follow up of the successful PROBA-1 program, in orbit since October 2001 . The spacecraft is not
SWAP will perform as an operational solar monitoring tool for space weather forecasting while it will also demonstrate new technological solutions: CMOS/APS detector, new off-axis telescope design, athermal structure, ...

LYRA (LYman-alpha RAdiometer) is a small compact solar VUV radiometer. This instrument is designed, manufactured and calibrated by a Belgian-Swiss-German consertium. It will monitor the solar flux in four UV passbands. The spectral channels bave been carefully selected for their relevance to space weather, solar physics and aeronomy, ranging from $1 \mathrm{~nm}$ to 220 nm. On the technological side, L.YRA will benefil from the pioneering UV detectors program using diamond technology. The LYRA data will produce valuable solar monitoring information, for operational space weather nowcasting and research.

This paper will detail the instrument concepts and their preparation for delivery to the platform.

larger than a domestic washing machine with a weight of $100 \mathrm{~kg}$. It will be launched as a piggy back payload, to reach a heliosynchrone polar orbit stabilized at 06:00.

PROBA-2 is developed under an ESA General Support Technology Program (GSTP) contract by a consortium led by Belgian industry. It has two main mission objectives:

- perform an in-flight demonstration of a series of new spacecraft technologies;

- support a scientific mission of a set of selected instruments.

As a technology evolution of PROBA-1 [1] successfully in orbit since October 2001 , the performances and the autonomous functions previously demonstrated by PROBA-1 will also be met or exceeded. This new platform will offer increased miniaturization and integration of avionics, improved spacecraft performances and additional technology 
demonstration in the field of power system and propulsion, providing thus more resources to the payloads.

PROBA-2 will be launched in 2007 together with the SMOS spacecraft. The spacecraft will permanently orient its top face towards the Sun, allowing quasi-interrupted solar observations, besides the eclipse seasons.

\section{if. PROBA-2 PAYLOAD}

SWAP and LYRA are the major components of the PROBA-2 scientific payload [2], both developed under the management of Centre Spatial de Liège (CSL, Belgium). SWAP will image the extreme UV solar corona with a dedicated telescope, while the LYRA radiometer will record the total solar radiation in four UV wavelength ranges.

The Academy of Sciences of the Czech Republic is developing the other components of the scientific payload:

- the Thermal Plasma Measurement Unit (TPMU) to evaluate the density and temperature of the ions, the Dual Segmented Langmuir Probe (DSLP).

These 4 instruments are developed in the frame of the PRODEX/ESA program.

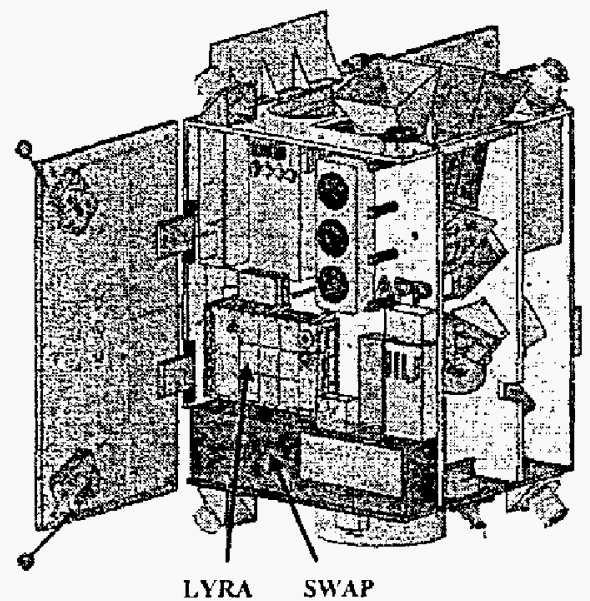

Figure 2. Open view of the PROBA.2 platform with SWAP and LYRA payload (couresy Verhaert N.V.)

\section{SWAP INSTRUMENT}

\section{A. Scientific Objectives}

SWAP [3] has been proposed as a successor of the Extreme ultraviolet Imaging Telescope [4] (EIT) onboard the joint ESA-NASA mission SOHO. The latter monitors the solar corona since 1996. The EIT instrument has proved to be particularly useful for space weather monitoring, thanks to its 'CME (Coronal Mass Ejections) watch program' providing an EUV image every 15 min.

Using techniques of solar observation developed by scientists in Belgium, for ESA's Space Weather project, SWAP and LYRA will give early warnings of etuptions on the Sun that provoke stormy weather throughout the Solar System. These often damage satellites and engineering systems on the Earth. Proba-2 itself will perform much of the analysis needed to spot the solar storms.

SWAP will provide observations centered on the $17.4 \mathrm{~nm}$ emission line. It will give indeed a particularly broad view on the 'solar weather'. Single $17.4 \mathrm{~nm}$ images are expected to give important information on the location of coronal holes (the source of high speed solar wind streams), active regions (potential source of solar flares) and filaments (potential eruption sites). Following the temporal evolution of these features as they rotate, gives additional important inputs to space weather forecasters.

SWAP will continue the systematic CME watch program of the aging EIT instrument, in a neighbor wavelength, that shall guarantee the detection of space weather related sotar events. Most of these significant events last on the order of $30 \mathrm{~min}$ to $45 \mathrm{~min}$. The SWAP time cadence will be of the order of ! minute, which is well adapted to record the occurrence of these events. Thanks to this high image cadence, SWAP will be a solar moniter capable of recording the time-evolution of every event in the low solar corona that might be of relevance wo the space weather.

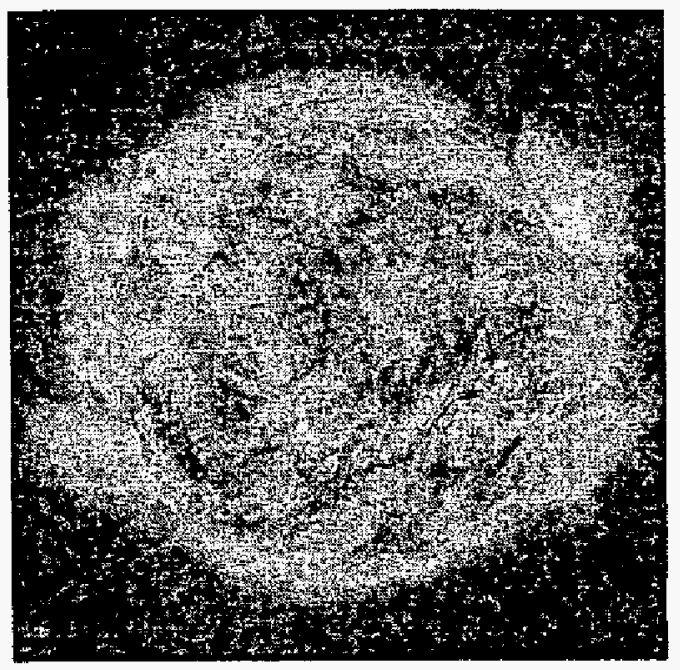

Figure 3. The EUV solar corona seen by SOHO/EIT, It is a realistic preview of the images that wil] be produced with SWAP, that will have a slightly larger field of view ( 54 aremin instead of 45 for EIT)

Using the PROBA platform agility. the on-board detection of CME will be used to trigger off-pointings in order to follow and image the CME leaving the Sun.

\section{B. Instrument Design}

The optics and focal plane assemblies of the SWAP telescope will be mounted on an optical bench designed to provide mechanical stability and insensitivity to thermal variations. With lightweight Invar structure, a stability better than $50 \mu \mathrm{m}$ will remain between the 2 mirrors in all the temperature range encountered by SWAP on-board PROBA-2.

SWAP is using a set of flexible mounts for attachment on 
the spacecraft to avoid platform perturbations and keep a stable optical bench.

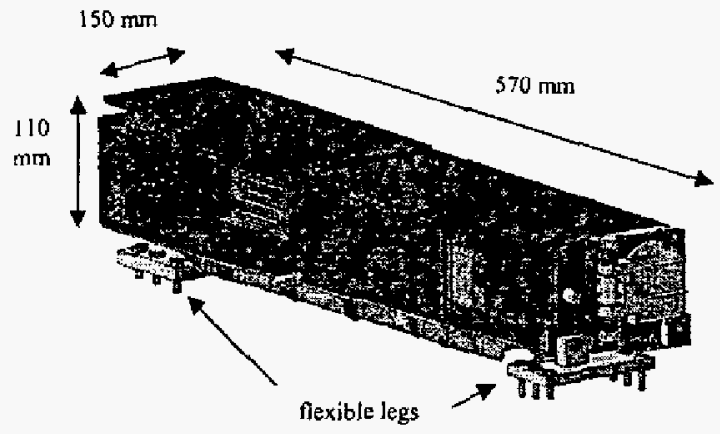

Figure 4. SWAP sensor box, with its main dimensions.

The instrument is encased in a lightweight housing (Fig. 4) equipped with an aperture mechanism to preserve the optical cavity from extemal contamination during on-ground activities and launch phase.

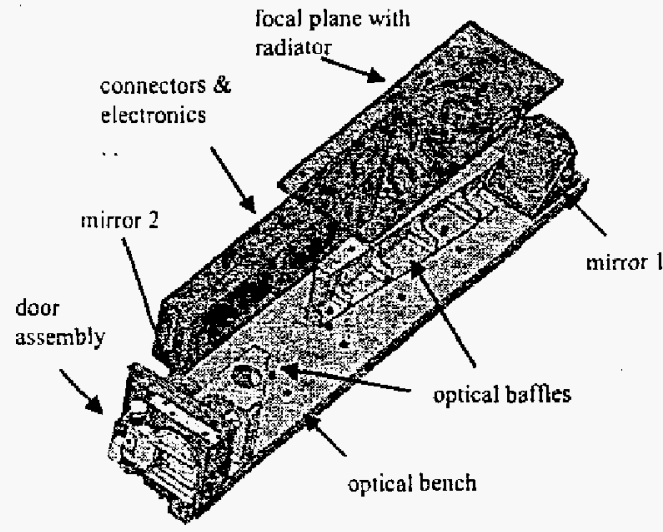

Figure 5. SWAP optical betch with its subsystems: the 2 mirrors, the optical bench, the focal planc assembly and the optical bafles. An aperure mechanism stands at the entrance of the instrument for cleanliness and filter protection on-ground and during launch.

The SWAP structure is designed using standard finite element analyses (Fig. 6) in order to optimize the mass and the stiffness with the high stability requirement. It will be qualified with a structural model before the flight model manufacturing,

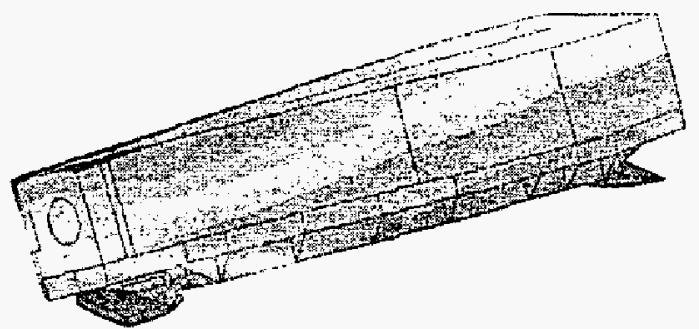

Figure 6. SWAP structure dynamic analysis showing one of the first global vibration modes (colors scale corresponding to absolute displacements, the feet being hardmounted)

The optical scheme is a novel off-axis Ritchey Chrétien scheme, that will offer numerous advantages for SWAP: (i) smaller and lighter primary mirror (no central obscuration); (ii) smaller aluminum foil filter thus reduced risk of mechanical damages; (iii) easy and efficient baffling system.

The off-axis telescope is illustrated in Fig. 7. A detailed description of the optical concept can be found in [5].

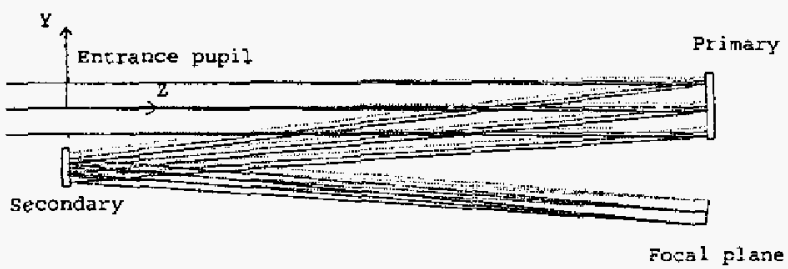

Figure 7. SWAP optical scheme

The optics (Fig. 8 ) will be manufactured and polished with a microroughness below $0.5 \mathrm{~nm}$ by AMOS S.A. (Belgium); then glued in dedicated mounts, and coated by Institut d'Optique Théorique et Appliquée (IOTA, France)

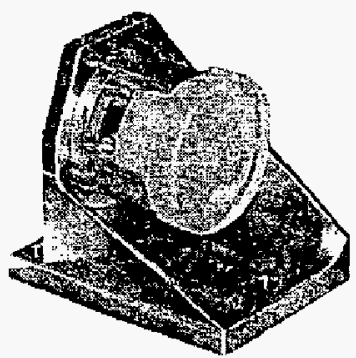

Figure 8. SWAP mirror concept, view of a protatype model (courtesy AMOS S.A.)

The optical coating is a multilayer composed of an altemance of $\sim 30$ layers of 2 different materials [6]. The overall stack is specifically designed to provide reflectivity in the extreme ultraviolet range and to achieve the spectral selection in a narrow band pass $(1.5 \mathrm{~nm})$ centered on $17.4 \mathrm{~nm}$ (Fig. 9). 


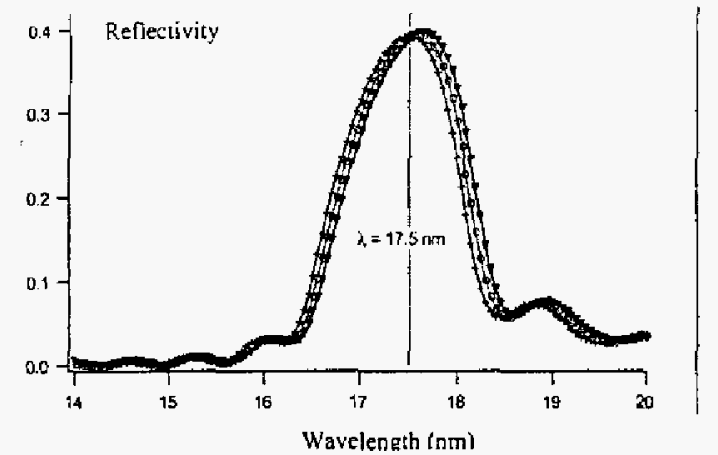

Figure 9. Refectivity provided by the multilayer coating in the extreme UV range. The bandpass is $-15 \mathrm{~nm}$, with peak reflectivity of $40 \%$. The differeni plots correspond to the different incident angles. (source: IOTA)

The focal plane will include the CMOS APS detector [7] (Fig. 11) from Fill factory N.V. (Belgium), and a dedicated cold finger connected to an external radiator, viewing the cold space for passive cooling down to $-20^{\circ} \mathrm{C}$. It is under manufacturing at OIP N.V. (Belgium). The detector being permanently cooled, it will trap potential contaminants released by the outgassing of the instrument surfaces in space, even with the high cleanliness level of SWAP. A decontamination heater has been implemented in order to release these contaminants that are very critical for EUV instruments. Periodic bake out sequences are planned in the SWAP operation plan.

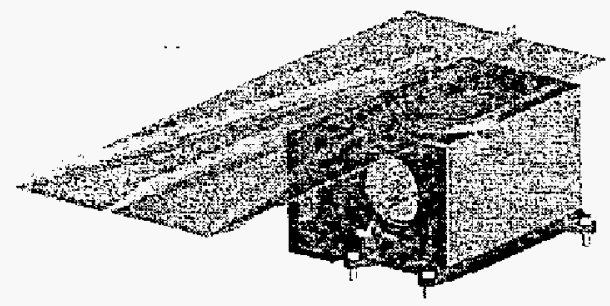

Figure 10. Focal plane assembly with its cooling radiator. (courtesy OIP N.V.)

The focal plane assembly (Fig. 10) will also include the proximity electronics required to readout the sensor, and drive the calibration diodes. The proximity electronics is directly connected to a specific memory compression and packetization module (MCPM) directly plugged in the central computer of PROBA-2 (ADPMS). The MCPM and the proximity electronics are developed by Deltatec S.A. (Belgium). A dedicated software running in the ADPMS will manage the SWAP data, with a compression module and a set of automatic event detection algorithms and data processing (cosmic ray removal, weak pixel compensation, ...).

SWAP and LYRA share the same power supply unit, named Interface Instrument Unit (IIU). This unit will be mounted on the instrument panel berween the 2 instruments. The IIU is built by Alcatel/Etca (Belgium).

The spacecraft resources are very limited. SWAP mass is limited to $10 \mathrm{~kg}$, with a mean power dissipation lower than 5
W. These 2 constrains are very demanding and required a deep optimization of the electronics as well as a lightweight mechanical structure.

The assembly, integration and all the qualification tests will be performed in the facilities of CSL. The optical calibration with EUV radiation is performed in Germany (PTB Bessy). SWAP is expected to be delivered to the platform in the first half of 2006.

The calibration of test detectors has already produced promising results (see Fig. 11).
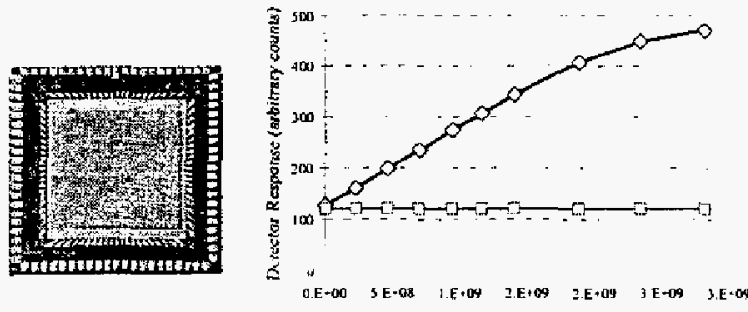

$$
\text { EUV incideral photers }
$$

Figure 11. SWAP test detector with scintillation coasing (left) and (righi) the linearity check (O) with EUV photons, until reaching saruration. The horizontal plot ( $\square$ ) is the dark leve!.

Similar tests will be performed with the optics alone, and with the fully integrated flight instrument in order to derive the final calibration of SWAP images.

\section{LYRA INSTRUMENT}

\section{A. Scientific Objectives}

The LYman-alpha Radiometer (LYRA) [8] [9], a smal] compact solar VUV radiometer accompanies SWAP. LYRA. is designed, manufactured and calibrated by a Belgian-SwissGerman consortium led by CSL, with ROB being the principal investigator.

Absolute measurements of the UV Sun irradiance are reputed to be difficult. They always require a space-born instrument. LYRA will be complementary to existing radiometers such as UARS, SEE/TIMED, SORCE, ... by monitoring the solar flux in four carefully selected UV passbands. The channels have been chosen for their relevance to aeronomy, space weather and solar physics: 1/ Lymanalpha $(121.6 \mathrm{~nm}), 2 /$ the $200-220 \mathrm{~nm}$ Herzberg continuum range (interference filters for the two former passbands), $3 / \mathrm{Al}$ filter channels (17-70 nm) covering lHe II-30.4 nm, 4/ XUV $Z_{r}$ channels ( $\left.1-20 \mathrm{~nm}\right)$, where solar variability is highest.

The LYRA data will produce valuable solar monitoring information, for operational space weather nowcasting and research. Similarly to SWAP, the LYRA science and operations will be managed by the solar team of the Royal Observatory of Belgium (ROB), as principal investigator. -

\section{B. Instizment Concept}

On the technological side, LYRA will benefit from diamond detectors: it will be the first space assessment of the pioneering UV detectors program, originally initiated at the 
ROB. Diamond, the wide bandgap material, makes the sensors radiation-hard and "solar-blind", which allows suppressing the usual filters, that block the unwanted visible, but attenuate seriously the desired UV radiation.

LYRA has a set of 3 redundant heads, each including the 4 spectral channels. Each channel corresponds to a collimator and a detector head (one detector, one filter, two calibration LEDs and a calibrated hole). The design of the heads takes also into account opening angle, cleanliness, and thermal issues. The LYRA optical design by PhysikalischMeteorologisches Observatorium Davos (PMOD, Swizterland) stems from the photometers of VIRGO onboard SOHO []0].

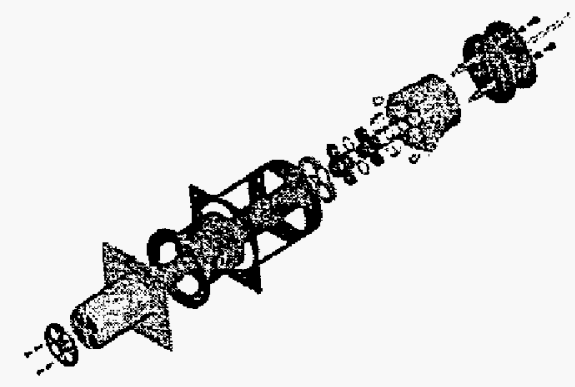

Figure 12. A single L, YRA head with 4 spectra! channels (radiation is coming from lower left side)

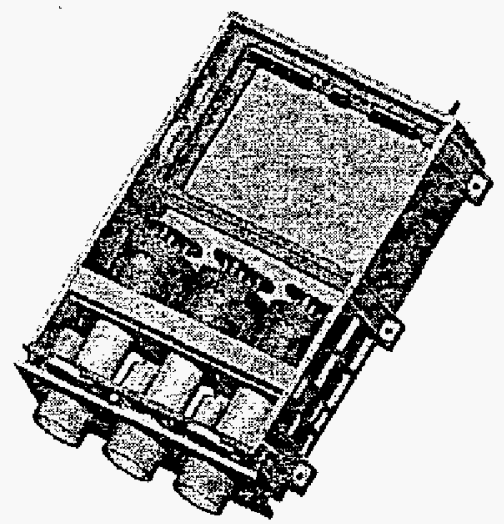

Figure 13. Open view of LYRA, without electronies. The 3 heads, each with 4 channels, are aligned each behind an aperture door (not shown).

The LYRA instrument has been optimized in order to match the very strict platform requirements in terms of mass, power and thermal range. The overall mass of L.YRA does not exceed $5 \mathrm{~kg}$, its power is limited to $2.7 \mathrm{~W}$ and it can be operated in a $\left[-40^{\circ} \mathrm{C},+60^{\circ} \mathrm{C}\right]$ temperature range.

\section{Detectors and fihers}

The LYRA solar blind diamond detectors are designed and fabricated at IMOMEC, Belgium with the collaboration of the National Institute for Materials Science (NIMS), Tsukuba, Japan and the Institut d'Electronique et de Microélectronique du Nord (IEMN), Liile, France. Two types of detectors are developed:

- MSM (photoconductor with Metal-Semiconductor-Metal junction);

- $\quad$ PiN (photodiod with $\mathrm{p}-\mathrm{i}-\mathrm{n}$ junction).

Detectors based on CDV diamond consist of a thin epitaxial CVD diamond layer or an epitaxial multilayer structure deposited on a natural Ila or synthetic HPHT diamond substrate.

CVD diamond PN junction and photoresistor have been optimized by mathematical modelling. The detector optimization included the improvement of the circular contact geometry, the photolithography process, the contact by laser and thermal annealing and the measurement of material and device parameters.

Based on the various data sets gathered during the calibration campaigns (Fig. 14), the PiN diodes show good response in the Herzberg channel but are insensitive at the Lyman alpha wavelength.

The MSM structures show higher responses with a solar blindness of typically 5 decades in magnitude between 200 and $400 \mathrm{~nm}$. As for the PiN detectors, the responsivity drops by a factor 10 in Lyman alpha spectral region. The MSM are selected for the Lyman alpha channel, where a signal of 100 pA is expected and is manageable by the LYRA electronic. Alternative detectors are considered to provide a spare or a calibtation backup in this channel. MSM with improved lithography mask or possibly Si detectors will equip the third L.YRA head.

Concerning the behavior in the XUV region, calibration tests performed at PTB Bessy synchrotron give the preference to MSM detectors.

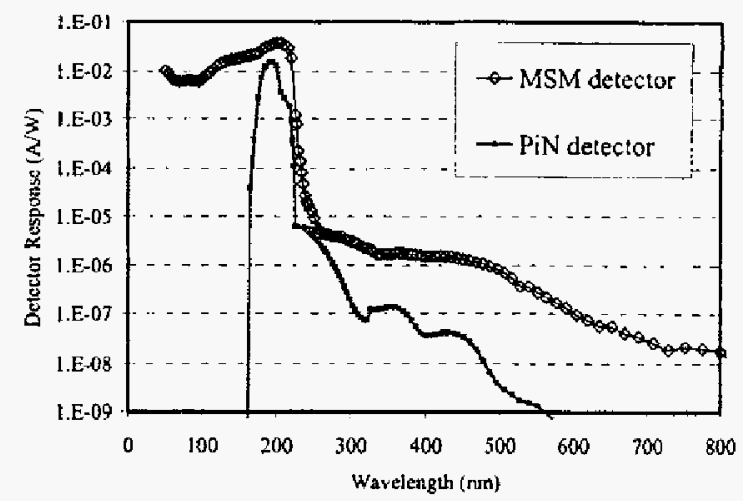

Figure 14. Typical responsivities measured for $\mathrm{PiN}$-diamond and MSM detectors

TABLE I

LYRA FILTER SELECTION

\begin{tabular}{|c|c|}
\hline Channel & Selected filter \\
\hline Lyman $\alpha 121.6 \mathrm{~nm}$ & 1 interferential filter on $\mathrm{MgF}_{2}$ \\
\hline Herzberg $200-220 \mathrm{~mm}$ & 1 interferential filter or M1 \\
\hline EUV $1: 17-70 \mathrm{~nm}$ & I Al foil filter on grid \\
\hline EUV 2: $1-20 \mathrm{~nm}$ & I $\mathrm{Zr}$ foil filter on grid \\
\hline
\end{tabular}


A dedicated spectral filter and calibration LEDs complement each channel. The different filters are summarized in Table I.

The redundancy strategy uses 2 redundant heads. One is used on a weekly basis, while the other remains closed most of the time and is only used on a monthly basis. This will give all the relevant data to evaluate the aging of the sensors.

\section{Instrument development}

LYRA design benefits from the heritage of the VIRGO experiment. Major efforts have been spent to characterize the new detectors in the spectral ranges of each channel. The solar blindness was also verified, as well as the transmission of the various filters. All these data were analyzed and compiled in order to build a radiometric model of the instrument. This tool was used to select the most adequate detectors for each channel to provide a useful solar signal.

An engineering model of the LYRA instrument has been built and tested with a breadboard of the external power electronics (Fig. 15).

The assembly of the flight model will be completed in summer 2005. It will be followed by a full acceptance with vibrations and thermal vacuum tests. The complete instrument will undergo an end-to-end calibration.

EMC (electromagnetic compatibility) verifications will be conducted with the IIU and the SWAP instrument before integration on the PROBA-2 platform.

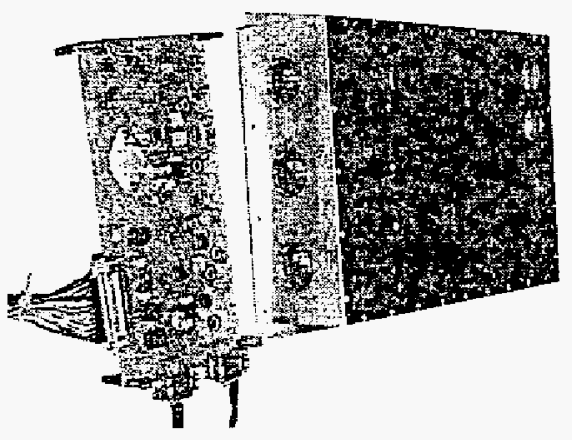

Figure 15. The engineering model of LYRA with its breadboard power electronics

\section{CONCIUSIONS}

The development of the PROBA-2 payload is in very good progress, on both the technological side and the mission preparation.

A new orbit has been obtained thanks to ESA, that will avoid eclipses of a 10:30 low earth orbit. This major improvement wiil really make LYRA and SWAF an operational space weather observatory. The solar payload has been designed to provide

- the demonstration of new technologies;

- valuable inputs for space weather forecasting;

- data for new scientific researches;

- a continuation to the solar survey of the aging SOHO mission;

- a support for the forthcoming SECCHJ/STEREO mission [11], to be launched in 2006.

SWAP and LYRA will contribute to demonstrating the feasibility of a technology that will be highly beneficial in the context of the Solar Orbiter ESA mission. New detectors, new compact optical scheme and on-board processing are key elements for this forthcoming ESA mission scheduled for the 2011-2013 time frame. This makes the scientific payload also a key element in the PROBA-2 demonstrator.

\section{ACKNOWLFDGMENTS}

The instrument teams wish to thank ESA for taking into consideration the scientific outcomes of the payload to select a new launcher to reach a 06:00 orbit.

The development of the SWAP instrument is funded by the Belgian Federal Science Policy Office (BELSPO), through the Belgium ESA/PRODEX program. LYRA is developed with the support of BELSPO, the Swiss Space Office (SSO) and the ESA/PRODEX program. SWAP and LYRA receive development support from their German Co-Investigators.

\section{REFERENCES}

[4] F Teston et al, "PROBA: ESA's Autoromy and Technology Demorstration Mission", Proceedings of the 13th Andual AIAAUSU: Conferente on Stmall Satcllites, August $23-26,1999$.

[2] P. Rochus, J.M. Defise et al PROBA-II Pazload: A Belgian Mini Space weather Observarory, IAF 2004.

13] D. Berghmans et al, SWAP on-board PROBA-2, a new EUV imager for solar rmanitoring, Submitted to Advances in Space Research, Oct 2004.

[4] JP. Delaboudinière et al, EIT: Exireme-UV imaging relescope for the SOHO mission; Solar Physies 162: 291-312, 1995.

[5] J.M. Defise et at, SWAP: Sum watcher with a new EUV telescope on a lechnology dertonstration plafform: ICSO Conferenee, 2004.

[6] JP Chauvineau et al, "Descriprion and performances of mirrors and multilayers for the extreme uilraviolet inaging telescope (EIT) of the SOHO mission". Proc. SPIE 1546, 576.

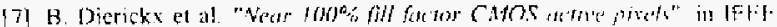
CCD \& AIS workshop, Brugge, Belgium, 5-7 june (1997); Proceedings p. P1.

[8] J.-F. Hochedez et al, LYRA : Melody instrument of Apollo Greek god of the Light and the Sw, SWENET Workshop (ESTEC) 3-5 Nov 2003.

[9] J.F. Hochedez et al, LYRA: the Solar UV radiometer aboard the ESA PROBA-2, Submitned in Oct 2004, accepted for publication in COSPAR. [10] Fröhisich et al, Solar Physics 162, p l01, 1995.

[11]J.D. Moses et al Sin-Earth connection coronal and heliospheric investigation (SECCHI) for the NASA STEREO mission. SPIE 4139-26, Jul 2000 . 